\title{
Anal cytological abnormalities and anal HPV infection in men with Centers for Disease Control group IV HIV disease
}

\author{
J M Palefsky, E A Holly, M L Ralston, S P Arthur, C J Hogeboom, T M Darragh
}

Objective: To characterise risk factors for abnormal anal cytology and anal human papilloma virus (HPV) infection in homosexual/bisexual men with advanced HIV related immunosuppression.

Design: Cross sectional study of men with Centers for Disease Control group IV HIV disease. Setting: The University of California San Francisco, AIDS Clinic.

Patients: 129 homosexual or bisexual men with group IV HIV disease.

Methods: A questionnaire was administered detailing tobacco, alcohol and recreational drug use, medical history, and sexual practices. Anal swabs for cytology and HPV studies were obtained, as was blood for CD4 levels.

Main outcome measures: Abnormal anal cytology and anal HPV infection.

Results: Abnormal anal cytology was detected in 39\% of subjects and anal HPV infection in $93 \%$ as measured by polymerase chain reaction (PCR). Risk factors for abnormal cytology in multivariate analysis included HPV $16 / 18$ infection (measured by PCR, RR $=2 \cdot 1,95 \% \mathrm{CI}=$ $1 \cdot 2-3 \cdot 5)$ and intravenous drug use $(\mathrm{RR}=1 \cdot 8,95 \% \mathrm{CI}=1 \cdot 2-2 \cdot 7)$. Infection with HPV 6/11 also had significantly elevated RRs in a separate model. Cigarette smoking, alcohol use, recre- $\mathrm{O}$ ational drug use, and low CD4 level were associated with abnormal anal cytology in univariate analysis, as was infection with multiple HPV types and high levels of hybrid capture group B viral DNA.

Conclusions: Anal cytological abnormalities and HPV infection are common among homosexual/bisexual men with group IV HIV disease. In this study population, the main risk factors for abnormal cytology were HPV infection and intravenous drug use.

(Genitourin Med 1997;73:174-180)

Keywords: anal intraepithelial neoplasia; anal cytology; human papilloma virus

\section{Introduction}

Before the human immunodeficiency virus (HIV) epidemic, the incidence of anal cancer was estimated to be approximately $35 / 100000$ among men with a history of receptive anal intercourse. ${ }^{1}$ This incidence is similar to that of cervical cancer in women before the introduction of cervical cytology screening and several times higher than current cervical cancer rates. $^{2}$ The past 20 years have seen an increase in the incidence of anal cancer in this group, although the degree to which HIV related immunosuppression has contributed to this increase is unclear. ${ }^{34}$

Much of the decrease in cervical cancer rates among women in the past few decades has been attributed to the introduction of routine screening for precancerous cervical disease. Detection of a lesion on cervical cytology, such as a high grade squamous intraepithelial lesion (HSIL), typically results in referral for colposcopy, colposcopy directed biopsy, and treatment of the lesion, and theoretically preventing progression of the lesion to invasive cancer.

There are many biological similarities between cervical and anal cancer ${ }^{5}$ that lend themselves to the use of anal cytology for detection and treatment of potentially precancerous anal disease. Among these similarities is a strong association with human papilloma virus (HPV) infection. ${ }^{5-7}$ As described in a recent review, several HPV types are known to infect the anogenital tract. HPV 16 and 18 are most strongly associated with HSIL and invasive cancer, whereas types $6,11,42,43$, or $44 \stackrel{0}{=}$ are primarily associated with low grade squamous intraepithelial lesions (LSIL), and are only rarely if ever detected in invasive cancers. ${ }^{8}$ HPV types 31, 33, 35, 45, 51, 52, and 56 are considered to have an "intermediate" risk of association with malignancy.

Previous studies have shown that anal squamous intraepithelial lesions (ASIL) are more o common among HIV positive men than $\tilde{O}$ among HIV negative men, especially among $\tilde{\omega}$ those HIV positive men with lower CD4 counts. ${ }^{9-12}$ Similarly, anal HPV infection is more common in HIV positive men with lower CD4 counts. ${ }^{11-15} \mathrm{HIV}$ related immunosuppression and infection with HPV 16 or 18 are predictive of HSIL development ${ }^{16}$ among men ${ }_{\Omega}$ followed prospectively. Lastly, recent data $\stackrel{\mathbb{Q}}{\stackrel{\mathrm{Q}}{2}}$ have shown that anal HPV infection and HPV related disease are more common in HIV positive women than in HIV negative women, and both are associated with lower CD4 levels in the HIV positive women. ${ }^{17}$

Other than HIV related immunosuppression and HPV infection, behavioural and biological risk factors for anal disease are poorly understood. A recent study conducted in Jamaica showed that seropositivity to human T cell leukaemia virus (HTLV)-1 was a pre- 
dictor for cervical HSIL in HIV negative women that was independent of HPV infection. ${ }^{18}$ The role of HTLV-1 infection as a cofactor for anal disease is unknown.

Currently there is no screening programme for anal disease in either men or women that is equivalent to cervical cytology screening. Further information about risk factors for anal disease to identify individuals who would most benefit from anal screening is of great importance. The goal of this study was to characterise behavioural and biological risk factors for abnormal anal cytology and anal HPV infection in a high risk group consisting of homosexual and bisexual men with Centers for Disease Control (CDC) group IV HIV disease.

\section{Materials and methods}

This study was conducted after approval from the University of California San Francisco Committee on Human Research. Study subjects were HIV positive with CDC group IV Disease. ${ }^{19}$ A total of 129 homosexual and bisexual men were recruited consecutively from the University of California San Francisco AIDS Clinic between 1990 and 1991. After informed consent was obtained, subjects were interviewed in detail about medical history: prescription drug use; exposure to diagnostic and therapeutic $x$ rays; history of sexually transmitted diseases, intestinal parasitic diseases, and hepatitis; anal conditions such as fissures and fistulas; and AIDS related symptoms and diseases. Subjects also were questioned about behavioural factors: tobacco, alcohol, and recreational drug use; and sexual practices. A thorough anal examination was then conducted that included insertion of a Dacron swab for anal cytology, as described elsewhere, ${ }^{10}$ followed by a swab for HPV DNA detection.

\section{ANAL CYTOLOGY}

Anal cytology was interpreted without knowledge of the clinical status of the subjects or their HPV DNA results. Anal cytological results were classified as normal, atypical squamous cells of uncertain significance (ASCUS), LSIL, or HSIL, using current criteria for evaluation of cervical cytology, as described previously. ${ }^{10}$

\section{HPV DNA DETECTION}

In this study, two different methods of HPV detection, polymerase chain reaction (PCR) and hybrid capture (HC) (Digene Diagnostics Inc, Silver Spring, MD, USA) were used. PCR is an amplification based test that is highly sensitive and detects low level HPV infection, but does not discriminate between low level and high level infection. HC is a semiquantitative, non-amplification based test that detects high level infection only. HC results are expressed as a relative light unit (RLU) ratio, calculated by dividing the chemiluminescent signal of the test sample by that obtained with a control sample containing 10 $\mathrm{pg} / \mathrm{ml}$ of HPV 16 DNA. The magnitude of the
RLU ratio increases with increasing quantity of HPV DNA in the specimen. HPV infection was classified as negative if the RLU ratio was less than 1.0 or positive if the ratio was greater than $1 \cdot 0$.

To obtain specimens for HPV DNA testing, an anal swab was inserted into the anal canal to collect exfoliated anal and rectal cells. The swab was then inserted into a tube containing 1 $\mathrm{ml}$ of sample transport medium (STM, Digene Diagnostics, Inc, Silver Spring, $M D$, USA) and frozen at $-70^{\circ} \mathrm{C}$ until analysis. After defrosting, each specimen was incubated for 1 hour at $55^{\circ} \mathrm{C}$ to inactivate HIV. A volume of $250 \mu \mathrm{l}$ was used for PCR analysis and the remainder was used for $H C$.

PCR was conducted using the MY09/MY11 HPV L1 consensus primers for HPV DNA amplification as well as primers for amplification of the human $\beta$ globin gene. ${ }^{20}$ Specimens in STM underwent phenol:chloroform extraction, followed by isopropanol precipitation. After centrifugation and drying, the pellet was dissolved in $20 \mu \mathrm{l}$ of TRIS-EDTA $\mathrm{pH} 7 \cdot 6$. A volume of $2 \mu \mathrm{l}$ was added to a solution with a final volume of $100 \mu \mathrm{l}$ containing $500 \mathrm{nM}$ of each primer, $200 \mathrm{mM}$ dNTPs, $2 \cdot 5$ units Taq polymerase, $50 \mathrm{mM} \mathrm{KCl,} 4 \mathrm{mM}$ $\mathrm{MgCl}_{2}$, and $10 \mathrm{mM}$ TRIS $\mathrm{pH} \mathrm{8.5.} \mathrm{After} 30$ cycles of amplification, $2 \mu \mathrm{l}$ of amplification mixture were applied to a nylon membrane and probed with a ${ }^{32} \mathrm{P}$ labelled HPV L1 consensus probe mixture. ${ }^{20} \mathrm{~A}$ separate membrane, prepared in a similar manner, was probed with a ${ }^{32} \mathrm{P}$ labelled probe to the human $\beta$ globin gene. Negative controls for each experiment consisted of amplification of solution containing all the above components except for the extract from the clinical specimens, as well as positive controls for the amplification of plasmid purified HPV DNA.

Specimens that were positive with the HPV consensus probe mixture were studied further to determine the specific HPV type by preparing membranes as described above with $2 \mu$ of specimen. The membranes were then probed with ${ }^{32} \mathrm{P}$ labelled probes to HPV $6,11,16,18$, $31,33,35,39,45,51$, and 52 . Specimens that were positive with the HPV consensus probe mixture but which were negative with all of the specific probes were considered to have an "unknown" HPV type. Specimens that showed no amplification of the $\beta$ globin gene were considered to be insufficient and were excluded from analysis.

$\mathrm{HC}$ was conducted according to the manufacturer's recommendations. Two separate tests were performed on each specimen, consisting of $100 \mu \mathrm{l}$ of specimen for detection of the low risk HPV types in probe group $A$ $(6 / 11 / 42 / 43 / 44)$ and $100 \mu l$ for detection of the intermediate and high risk HPV types in probe group B (16/18/31/33/35/45/51/52/56). The result of the test was expressed as the RLU ratio, determined by dividing the chemiluminescent signal of the test sample by that obtained with a control sample containing $10 \mathrm{pg} / \mathrm{ml}$ of HPV 16 DNA. Specimens with a RLU ratio of 1.0 or more were considered positive. Specimens positive with probe group 
Table 1 Cutpoints for categories used to quantify behavioural activities among $129 \mathrm{HIV}$ positive homosexual men in the San Francisco Bay area

\begin{tabular}{|c|c|c|c|c|}
\hline Exposure measure & 0 (Never, rare) & 1 (Low) & 2 (Medium) & 3 (High) \\
\hline $\begin{array}{l}\text { Usual number of drinks/day } \\
\text { Maximum drinks/day } \\
\text { Number of days/month drank } \\
\text { Number of cigarettes/day } \\
\text { Number of recreational drugs } \\
\text { Number of male partners in } 5 \text { years } \\
\text { Number of female partners in } 5 \text { years } \\
\text { Frequency of sexual activity } \\
\text { Percentage time engaged in activity }\end{array}$ & $\begin{array}{l}0-1 \\
0-2 \\
<1 \\
0 \\
0 \\
1-3 \\
0 \\
\text { Never } \\
\text { Never }\end{array}$ & $\begin{array}{l}2-4 \\
3-7 \\
1-8 \\
<15 \\
1 \\
4-49 \\
1 \\
1 / \text { month } \\
<50 \%\end{array}$ & $\begin{array}{l}5-7 \\
8-10 \\
9-20 \\
16-29 \\
2 \\
50-199 \\
2-4 \\
1 / \text { week } \\
50-99 \%\end{array}$ & $\begin{array}{l}\geqslant 8 \\
\geqslant 11 \\
\geqslant 21 \\
\geqslant 30 \\
\geqslant 3 \\
\geqslant 200 \\
\geqslant 5 \\
\geqslant 3 / \text { week } \\
100 \%\end{array}$ \\
\hline
\end{tabular}

^Drugs used by at lease $50 \%$ of subjects were marijuana, poppers, cocaine, LSD, speed, magic mushrooms, and ecstasy. Drugs used by less than $50 \%$ were methaqualone, angel dust, ethylchloride, valium, barbiturates, crack cocaine, heroin, diet pills, any drug? injected, and any drug used rectally.

A were retested separately for HPV 6/11 and $42 / 43 / 44$ if sufficient specimen remained using $50 \mu \mathrm{l}$ of specimen instead of the standard $100 \mu \mathrm{l}$ for the first round as described above. Specimens positive with probe group B were retested separately for HPV 16/18, 31/33/35 and $45 / 51 / 52 / 56$ using $50 \mu \mathrm{l}$ of specimen instead of the standard $100 \mu \mathrm{l}$ if sufficient specimen remained.

MEASUREMENT OF CD4 LEVELS AND ANTIBODIES TO HTLV-1

CD4 counts were measured using the Coulter
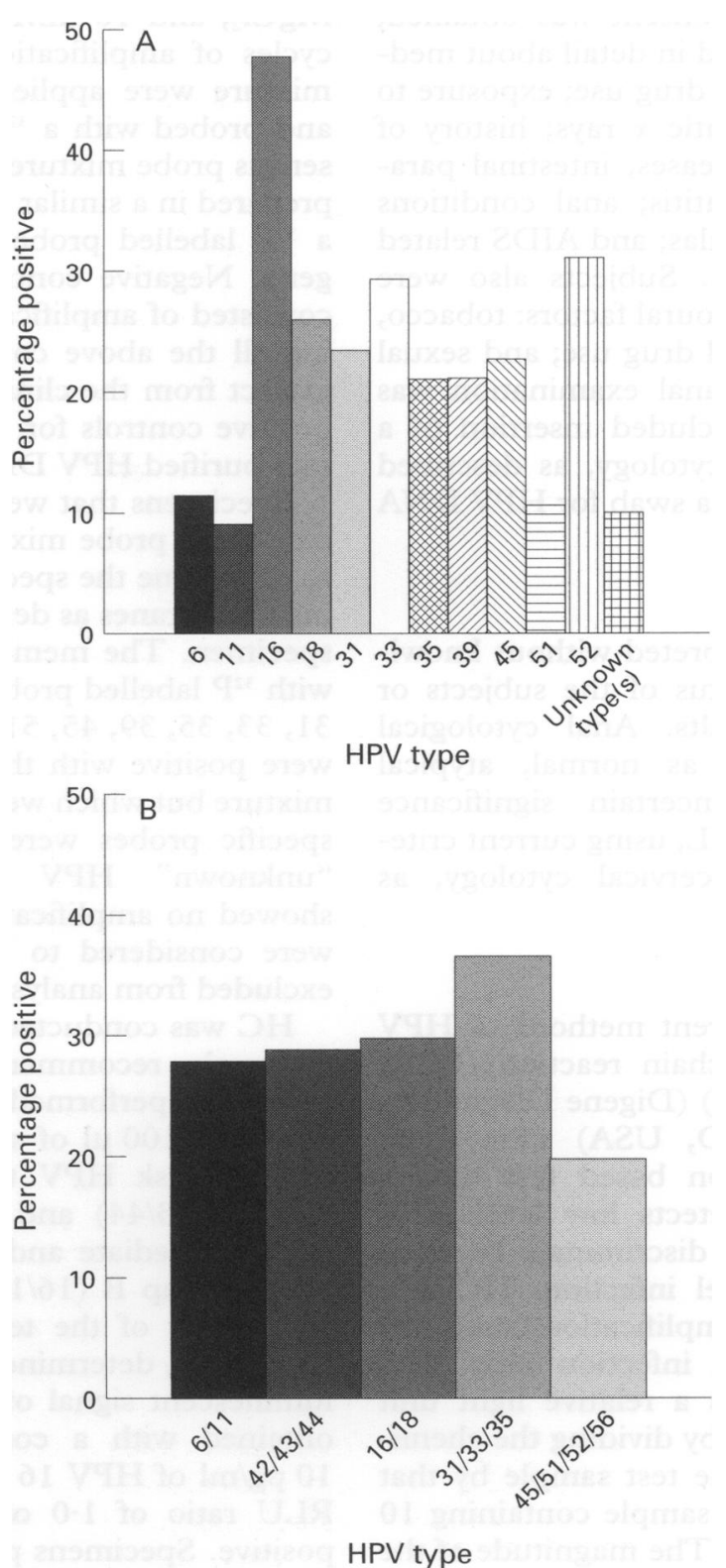

EPICS-Profile II flow cytometer. Plasma anti bodies to HTLV-1 were measured for all sub든 jects at baseline using an ELISA assay (Abbot HTLV-1 2.0 EIA, Abbott Laboratories Abbott Park, IL, USA).

STATISTICAL ANALYSIS

Summary variables quantifying lifetime expo sure were created because most subjects had sampled nearly all behavioural activities. Recreational drugs were divided into two groups - those used by at least $50 \%$ of the sub jects and those used by less than $50 \%$. Fon each group of drugs three scores were com? puted: (i) the number of drugs ever used, (ii) the number used at least once per week, anc (iii) the number used during multiple time periods. These six scores were then averagedo for an overall drug use score. For the other behavioural variables three measures of expoछ sure were ascertained to quantify exposure: (i the portion of relevant lifetime engaged in th activity, (ii) the usual amount of activity duro ing that period, and (iii) the peak amount of activity. The categories that comprise thes $\vec{E}$ exposure measures are shown in table 1 ?. Different measures of duration and quantity of activity were used depending upon the activ ity. The derived variables were converted to scores of $0,1,2$, and 3 with larger numbers representing higher levels of exposure. These scores were averaged to create a summary vari able for each behaviour for each individual The cutpoints for these scores were deter? mined from the combined distributions of variables measured similarly, such as the fre quency of various sexual activities. The sum? mary risk factor exposure categories created by this process were used to divide study subject 8 into the following exposure groups: (1) nevero rare: men were exposed to the risk factor over $\mathscr{Q}$ small portion of their lifetime and never of rarely engaged in the activity; (2) low expo sure: men who exceeded the limits of the never, rare category in at least one aspect of the activity; (3) moderate exposure: men who engaged in the activity most of their lives for moderate amount of time and may have occa sionally engaged in it extensively; and (4) hige exposure: men who engaged in the activit most of their lives and usually engaged in extensively.

The SAS statistical package was used for data analysis. Relative risks (RR) for developing abnormal cytology, $95 \%$ confidence inter-
Figure 1(A) Prevalence of specific human papillomavirus (HPV) types in 118 anal swab specimens using poly
chain reaction. (B) Prevalence of specific HPV groups in 109 anal specimens using hybrid capture. 
Table 2 Association between hybrid capture (HC) positivity, relative light unit (RLU) ratio ${ }^{\star}$, and abnormal anal cytology

\begin{tabular}{|c|c|c|c|}
\hline & $\begin{array}{l}\text { Abnormal } \\
\text { cytology }\end{array}$ & $\begin{array}{l}\text { Normal } \\
\text { cytology }\end{array}$ & $R R(95 \% C I)$ \\
\hline \multicolumn{4}{|l|}{ HC result: } \\
\hline $\begin{array}{l}\mathbf{A}(-) \mathbf{B}(-) \\
\mathbf{A}(+) \mathbf{B}(-)\end{array}$ & $0\}$ & $20\}$ & $1 \cdot 0 \dagger$ \\
\hline $\mathbf{A}(-) \mathbf{B}(+)$ & 14 & 22 & $5 \cdot 4(1 \cdot 3-23)$ \\
\hline \multirow{2}{*}{\multicolumn{4}{|c|}{ HC group A RLU ratio: }} \\
\hline & & & \\
\hline Negative, $<1.0$ & 14 & 42 & 1.0 \\
\hline $1-10$ & 13 & 13 & $2 \cdot 0(1 \cdot 1-3 \cdot 6)$ \\
\hline $10-100$ & 16 & 19 & $1 \cdot 8(1 \cdot 0-3 \cdot 3)$ \\
\hline$>100$ & 5 & 1 & $3.3(1.9-5.9)$ \\
\hline \multicolumn{4}{|c|}{ HC group B RLU ratio: } \\
\hline Negative, $<1.0$ & 2 & 26 & $1 \cdot 0$ \\
\hline $1-10$ & 13 & 20 & $5.5(1.4-22)$ \\
\hline $10-100$ & 25 & 26 & $6 \cdot 9(1 \cdot 8-27)$ \\
\hline$>100$ & 8 & 3 & $10 \cdot 2(2 \cdot 5-41)$ \\
\hline
\end{tabular}

${ }^{\star}$ Defined as the ratio of the chemiluminescent signal in the test sample divided by that of the control containing $10 \mathrm{pg} / \mathrm{ml}$ of viral DNA.

tFor reference group, $\mathrm{A}(-) \mathrm{B}(-)$ and $\mathrm{A}(+) \mathrm{B}(-)$ were combined.

vals (CI), Fisher's exact test, and the $\chi^{2}$ test for association were calculated for risk factors recorded as present or absent, primarily medical conditions, and treatments. The MantelHaenszel $\chi^{2}$ test for linear trend was computed

Table 3 Univariate RRs for abnormal anal cytology by CD4 levels and history of medical conditions, substance use, and sexual practice*

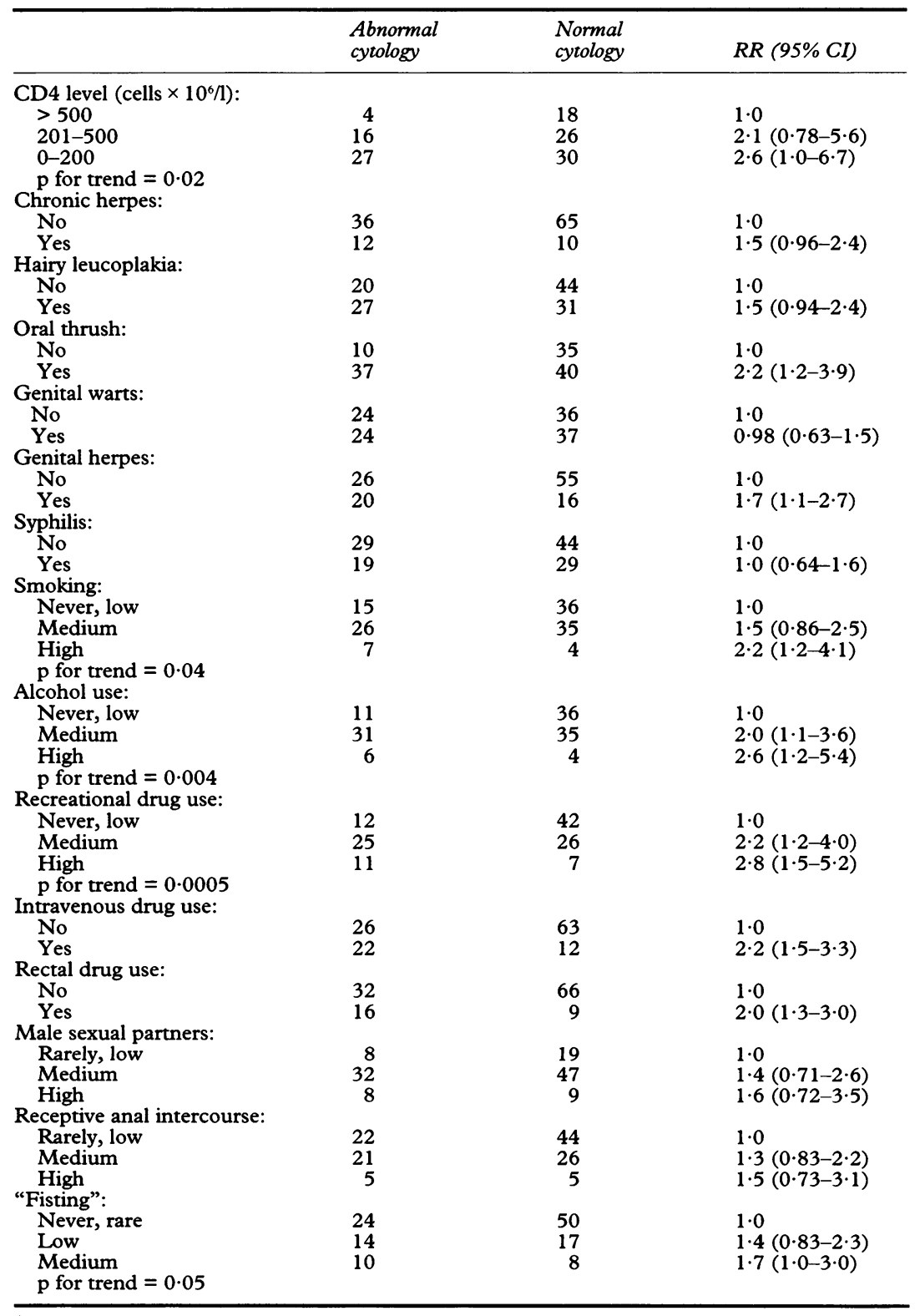

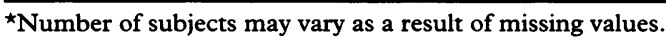

for risk factors with multiple levels of exposure when appropriate. Stepwise logistic regression was used to determine the relative strengths of association between abnormal cytology and the various risk factors with adjustment for other factors of interest. Finally, the MantelHaenszel procedure was used to compute adjusted relative risks.

\section{Results}

POPULATION DEMOGRAPHICS

A total of 129 men were enrolled in this study and all were HIV positive by ELISA and western blot tests. Three subjects had cytology specimens that were insufficient for interpretation and three had missing results, leaving 123 subjects available for the laboratory related analyses. The mean age of the study population was 41 years (range 24-66) and was $88 \%$ white, $5 \%$ Hispanic, $4 \%$ African-American, $2 \%$ American Indian, and $1 \%$ Asian or Pacific Islander. The median income level at the time of study entry was between $\$ 10000$ and $\$ 20000$ (range $<\$ 10000$ to $>\$ 100000$ ) and the median number of years of schooling was 14 (range 6 to $>16$ ).

PREVALENCE OF ABNORMAL ANAL CYTOLOGY AND ANAL HPV INFECTION

Of 123 anal cytology smears suitable for interpretation, 48 were abnormal (39\%) and 75 (61\%) were normal. Of the 48 abnormal smears, 35 were ASCUS, 12 were LSIL, and one was HSIL. The results of HPV PCR testing are shown in figure 1 (A). PCR results were available for 118 subjects, 113 of whom also had data on cytological diagnosis. One hundred and ten (93\%) were positive for at least one type of HPV. HPV 16 was the most commonly detected HPV type, followed by HPV 52 and HPV 33. Twelve (10\%) specimens contained unknown types only. Infection with more than one HPV type was detected in 97 of $118(82 \%)$ of samples analysed.

The results of $\mathrm{HC}$ testing are shown in figure 1(B). HC results were available for 129 subjects, 123 of whom also had data on cytological diagnosis. In all, 109 of 129 (85\%) subjects were positive using $\mathrm{HC}$. The most common group detected was HPV 31/33/35, followed by HPV $16 / 18$. Infection with more than one HC group was detected in 43 of 113 samples (38\%) with sufficient volume for analysis of all groups.

RISK FACTORS FOR ABNORMAL ANAL CYTOLOGY In this study, there were high rates of HPV positivity using both PCR and HC. Because many subjects had multiple types, "type specific" analyses should be interpreted cautiously. Overall, HPV infection using PCR was associated with abnormal cytology $(p=0.04$, Fisher's exact test). Each of the seven subjects who was PCR negative and the 11 subjects who had only unknown HPV types had normal anal cytology. The RR for abnormal cytology measured by PCR was separately calculated for HPV 6/11 $(\mathrm{RR}=1.5, \mathrm{CI}=$ $1 \cdot 0-2 \cdot 4), 16 / 18(\mathrm{RR}=2 \cdot 5, \mathrm{CI}=1 \cdot 4-4 \cdot 5)$, 
Table 4 Mutually adjusted RR for human papilloma virus (HPV) infection and intravenous drug use for abnormal anal cytology*

\begin{tabular}{ll}
\hline Risk factor & Adjusted $R R+(95 \%$ CI $)$ \\
\hline HPV 16/18 by PCR & $2 \cdot 1(1 \cdot 2-3 \cdot 5)$ \\
Intravenous drug use & $1 \cdot 8(1 \cdot 2-2 \cdot 7)$ \\
HPV 16/18 by HC & $1 \cdot 8(1 \cdot 2-2 \cdot 8)$ \\
Intravenous drug use & $1 \cdot 9(1 \cdot 3-2 \cdot 9)$ \\
HPV 6/11 by PCR & $1 \cdot 5(0 \cdot 97-2 \cdot 5)$ \\
Intravenous drug use & $2 \cdot 1(1 \cdot 4-3 \cdot 3)$ \\
HPV 6/11 by HC & $1 \cdot 8(1 \cdot 2-2 \cdot 8)$ \\
Intravenous drug use & $2 \cdot 2(1 \cdot 5-3 \cdot 4)$ \\
\hline
\end{tabular}

*HPV RR adjusted for intravenous drug use, intravenous drug use RR adjusted for HPV.

tReference group is absence of the factor of interest.

$31 / 33 / 35 \quad(\mathrm{RR}=1 \cdot 7, \quad \mathrm{CI}=1 \cdot 0-2 \cdot 8), \quad$ and $39 / 45 / 51 / 52(R R=1 \cdot 9, C I=1 \cdot 1-3 \cdot 2)$.

Overall, a positive HC result was associated with abnormal anal cytology $\left(\mathrm{p}<0.001, \chi^{2}\right)$. The RR for abnormal cytology using $\mathrm{HC}$ was elevated for all groups except HPV 42/43/44 $(\mathrm{RR}=1.2, \mathrm{CI}=0.73-1.9)$. The highest $\mathrm{RRs}$ were associated with $\mathrm{HPV} 31 / 33 / 35(\mathrm{RR}=$ $2 \cdot 6, \mathrm{CI}=1 \cdot 6-4 \cdot 2)$ and $16 / 18 \quad(\mathrm{RR}=2 \cdot 2$, $\mathrm{CI}=1 \cdot 4-3 \cdot 3)$, followed by $6 / 11(R R=1 \cdot 9$, $\mathrm{CI}=1 \cdot 3-2 \cdot 9)$ and $45 / 51 / 52 / 56 \quad(R R=1 \cdot 7$, $\mathrm{CI}=1 \cdot 1-2 \cdot 6)$. Infection with a group $\mathrm{B}$ type by $\mathrm{HC}$ was associated with a higher $R R$ for abnormal anal cytology than infection with a group A type; however, the CI for each of these groups were wide (table 2). The risk of abnormal anal cytology increased with higher HC RLU ratios for both group $A$ and $B$, with the highest risk associated with higher levels of group B (table 2).

Lifestyle factors and health status associated with abnormal anal cytology are presented in table 3. Lower CD4 counts and other markers of immune suppression such as oral thrush were significantly associated with abnormal anal cytology. Of the medications for AIDS associated illnesses examined, only trimethoprim-sulphamethoxazole $(\mathrm{RR}=1 \cdot 8,95 \% \mathrm{CI}$ $=1 \cdot 2-2 \cdot 8)$ was associated with abnormal anal cytology. Smoking, alcohol use, recreational, intravenous, and rectal drug use were each associated with abnormal anal cytology (table 3), with RRs increasing with higher categories of use.

Among the sexual behaviours studied, only "fisting" was significantly associated with abnormal anal cytology (table 3). Althoug果 RRs were elevated, no other sexual behaviouse were significantly associated with abnormat cytology. Among the sexually transmitted diş eases assessed, only a history of genital herpes was associated with abnormal anal cytology. ?

Logistic regression was used to measure the independent risks for exposures associateg with abnormal cytology. Included as possib predictors in the models were: HPV infection CD4 level, smoking, alcohol use, rectal drug use, intravenous drug use, number of femake and male sexual partners, receptive and insertive anal intercourse, fisting, "rimming"? and history of herpes simplex, warts, syphilis, gonorrhoea, or non-gonococcal urethritis Four separate models were run, one for each of the following measures of HPV infection HPV $16 / 18$ by HC; HPV $16 / 18$ by PCR; HPE 6/11 by HC; and HPV 6/11 by PCR. When HPV was in all four models without intra venous drug use, there was an association between abnormal cytology and several behav ioural risk factors. However, in all cases the association with intravenous drug use was the strongest (data not shown). HPV infection and intravenous drug use have similar RRs fof abnormal cytology after adjustment for each other (table 4). The adjusted and unadjusted RRs were essentially the same. After adjusting for HPV infection and intravenous drug use the other risk factors that had been associated with abnormal anal cytology in univariate analyses could not be evaluated owing to smat numbers in the subgroups.

Plasma antibodies to HTLV-1 were measured for all subjects. Only three of 129 (2\% subjects were positive for HTLV-1 and none of these subjects gave a history of intravenou

Table 5 RRs and 95\% CIs for risk factors for anal human papilloma virus (HPV) 16 or 18 or HPV 6 or 11 as detected by polymerase chain reaction (PCR) and hybrid capture (HC)

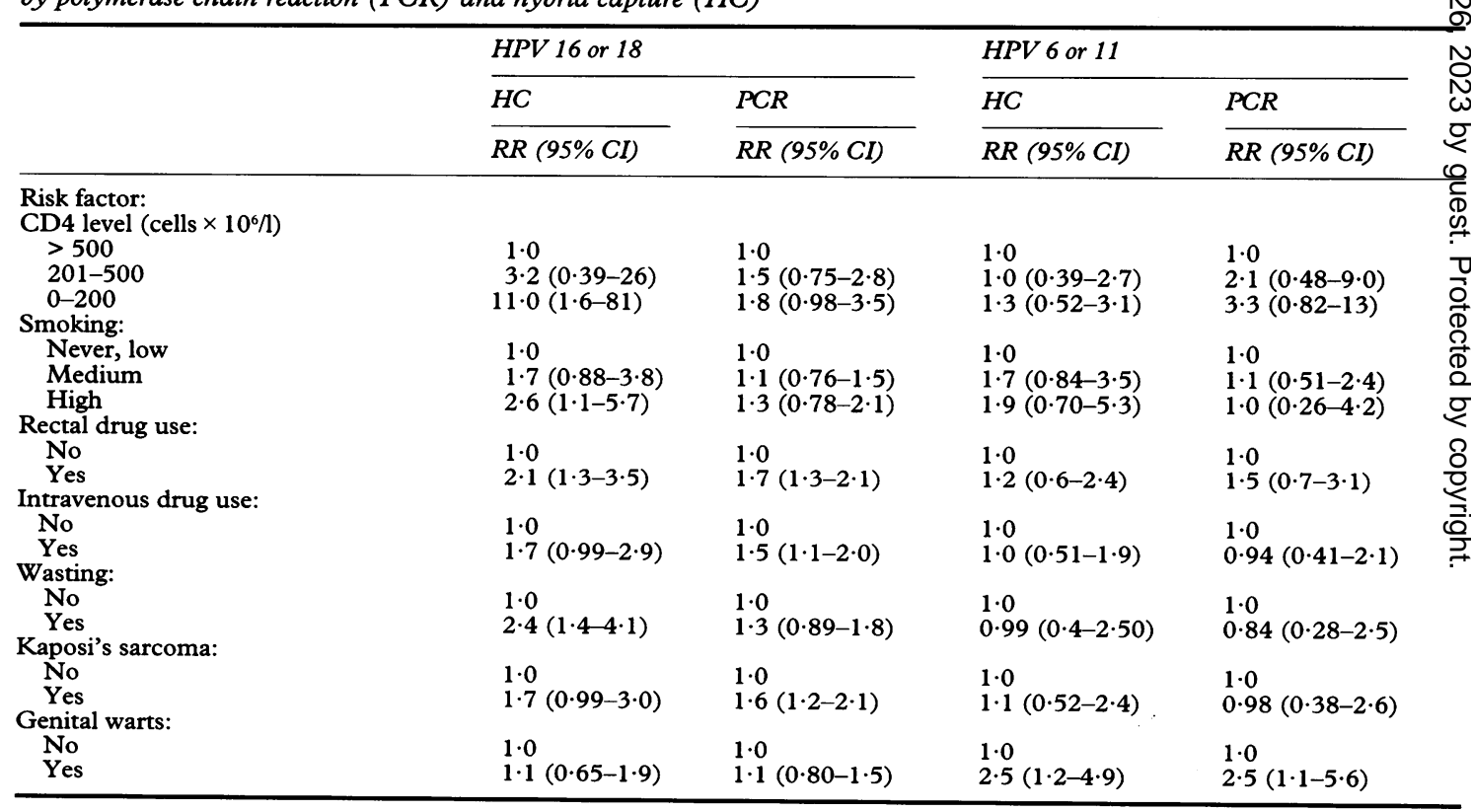


drug use.

RISK FACTORS FOR ANAL HPV INFECTION

To determine if risk factors for detection of high risk HPV types differed from those of low risk HPV types, risk factors similar to those described above for cytological abnormalities were tested separately for association with detection of HPV 16/18 and HPV 6/11 using PCR and HC (table 5). Some risk factors for HPV 16/18 differed from those associated with HPV 6/11. Using HC, factors associated in univariate analysis with HPV 16/18, but not HPV 6/11, included low CD4 count, rectal drug use, and wasting. HPV 16/18, but not HPV 6/11, was associated with a history of Kaposi's sarcoma and intravenous drug use using PCR (borderline significant with $\mathrm{HC}$ ). In contrast, HPV $6 / 11$ but not HPV $16 / 18$ was associated with a history of genital warts using either PCR or HC.

\section{Discussion}

Based on the similarity between cervical SIL and ASIL, a screening programme among high risk individuals may reduce the incidence of anal cancer in this group. In addition, ASIL is typically friable and bleeds easily with minor trauma, and there is a theoretical concern that HIV transmission may be potentiated between sexual partners when the anal receptive partner has ASIL. For both reasons, a carefully targeted screening programme may be of value to those at high risk of anal disease.

In this report, we characterised risk factors for anal disease and anal HPV infection among men at high risk - that is, those with CDC defined group IV HIV disease. Since the biological characteristics of this study population differ from an HIV negative population or a population that spans a wider spectrum of HIV disease, the findings of this study may not be generalisable to the general population of homosexual men.

In this group of men with advanced HIV related immunosuppression, anal cytological abnormalities were found in nearly $40 \%$ of the population, a level similar to that in an earlier study of a different group of men with symptomatic HIV disease. ${ }^{10} \mathrm{~A}$ high proportion of the abnormal cytologies were ASCUS in the current study. Justification for inclusion of ASCUS into the "abnormal" category includes data from previous work ${ }^{10}$ showing that the proportion of specimens with ASCUS containing HPV infection is similar to that with higher grades of cytological abnormality. In addition, approximately half of men with ASCUS on cytology have concurrent biopsy proved anal disease (J M Palefsky, unpublished data).

HPV infection was common in our study population using both PCR and HC. Our data showing $84 \%$ positivity using $\mathrm{HC}$ are similar to the $74 \%$ rate in men with AIDS/ARC reported in a recent study ${ }^{15}$ using the Virapap/Vira Type technique. Similar to earlier studies, low CD4 counts and HPV infection were associated with abnormal anal cytology in our study. This is especially significant given the low mean CD4 counts in our study population. Oral thrush, a clinical marker of advanced immunosuppression, was also associated with abnormal cytology. Concurrent infection with one or more HPV types from both the HC A and B groups was associated with the highest risk for abnormal cytology. Higher levels of HPV DNA as reflected by the HC RLU ratios showed increased risk, with higher RRs among the $B$ types.

Several lifestyle factors were associated with abnormal cytology, including smoking, alcohol, recreational and intravenous drug use. Of these, intravenous drug use was most strongly associated with abnormal cytology. After adjusting for intravenous drug use, exposure to other factors was not significantly associated with increased risk. Intravenous drug use may be a marker for other risky behavioural factors or may reflect a second infectious agent that is potentiating HPV associated anal disease. Based on data from Jamaica showing that HTLV-1 infection is associated with cervical HSIL even when adjusted for HPV infection, ${ }^{18}$ we sought to determine if intravenous drug use in our study was a marker for HTLV-1 infection. The low rate of HTLV-1 seropositivity suggests that it was not playing any role in our population.

Several studies have shown that the number of lifetime sexual partners is a risk factor for cervical SIL in women. ${ }^{21}{ }^{22}$ In our study, only fisting was associated with anal disease and may reflect high risk behaviour leading to increased risk of exposure to HPV. Because these men all had quite similar high risk behaviours for HIV and HPV acquisition, fine discrimination among various behaviours was not possible.

Examination of risk factors for anal HPV infection in this study was complicated by high rates of HPV positivity in the study population. However, comparison of risk factors for detection of high risk types such as HPV 16/18 and those of low risk types revealed several interesting differences. Detection of HPV 6/11 but not HPV 16/18 was associated with a history of anogenital warts. Conversely, detection of HPV $16 / 18$ but not HPV 6/11 was associated with signs and symptoms of more advanced immune suppression. Prospective studies will be needed to determine if this may be explained by higher rates of persistence of $16 / 18$ as immunosuppression increases.

In summary, our data show that a high proportion of HIV positive men with CDC group IV HIV disease have both anal cytological abnormalities and anal HPV infection. We show that, in this population, those at highest risk for anal abnormalities include men with anal HPV infection, particularly those positive for a group B HPV type using HC, and men with a history of intravenous drug use. These groups should be strongly considered as candidates for anal cytology screening to identify and treat potentially precancerous anal disease.

Supported by NIH grant R01CA54053, by funds provided by the Cigarette and Tobacco Surtax Fund of the State of 
California through the Tobacco Related Diseases Research Program of the University of California (grant RT484), and a grant from Digene Diagnostics, Inc. The authors wish to thank Shirley Koehler, PA, and Julia Jones and Jennifer Kristianson for their able assistance.

1 Daling JR, Weiss NS, Hislop TG, Maden C, Coates RJ, Sherman KJ, et al. Sexual practices, sexually transmitted diseases and the incidence of anal cancer. $N$ Engl $\mathcal{F} \mathrm{Med}$ 1987;317:973-7.

2 Qualters JR, Lee NC, Smith RA, Aubert RE. Breast and cervical cancer surveillance, United States, 1973-1987. $M M W R$ 1992;41:1-15.

3 Rabkin CS, Yellin F. Cancer incidence in a population with a high prevalence of infection with human immunodeficiency virus type 1. $\mathcal{F}$ Natl Cancer Inst 1994,86:1711-6.

4 Melbye M, Cote TR, Kessler L, Gail M, Biggar RJ. High incidence of anal cancer among AIDS patients. The AIDS/Cancer Working group. Lancet 1994;343:636-9.

5 Palefsky J. Anal cancer in HIV-positive individuals: an emerging problem. AIDS 1994:283-95.

6 Zaki SR, Judd R, Coffield LM, Greer P, Rolston F, Evat BL. Human papillomavirus infection and anal carcinoma. Retrospective analysis by in situ hybridization and the polymerase chain reaction. Am $\mathcal{F}$ Pathol 1992;140 1345-55.

7 Bosch FX, Manos MM, Munoz N, Sherman M, Jansen AM, Peto J, et al. Prevalence of human papillomavirus in cervical cancer: a worldwide perspective. International biological study on cervical cancer (IBSCC) Study group. F Natl Cancer Inst 1995;87:796-802.

8 Palefsky JM, Holly EA. Molecular virology and epidemiology of human papillomavirus and cervical cancer. Cancer ogy of human papillomavirus and cervical

9 Melbye M, Palefsky J, Gonzales J, Ryder LP, Nielsen $H$, Bergmann $\mathrm{O}$, et al. Immune status as a determinant of human papillomavirus detection and its association with anal epithelial abnormalities. Int $\mathcal{F}$ Cancer 1990;46:203-6.

10 Palefsky JM, Gonzales J, Greenblatt RM, Ahn DK Hollander $H$. Anal intraepithelial neoplasia and ana papillomavirus infection among homosexual males with group IV HIV disease. $\mathcal{F A M A}$ 1990;263:2911-6.

11 Kiviat NB, Critchlow CW, Holmes KK, Kuypers J, Sayer J, Dunphy C, et al. Association of anal dysplasia and human papillomavirus with immunosuppression and HIV infection among homosexual men. AIDS 1993;7: HIV

12 Palefsky JM, Shiboski S, Moss A. Risk factors for anal human papillomavirus infection and anal cytologic abnormalities in HIV-positive and HIV-negative homo-
sexual men. $f$ Acquir Immune Defic Syndr 1994;7: sexual men.

13 Caussy D, Goedert J, Palefsky J, Gonzales J, Rabkin CS, DiGiola RA, et al. Interaction of human immunodeficiency and papilloma viruses: association with anal epithelial abnormality in homosexual men. Int $\mathcal{F}$ Cancer 1990;46:214-9.

14 Critchlow CW, Holmes KK, Wood R, Krueger L, Dunphy C, Vernon DA, et al. Association of human immunodeficiency virus and anal human papillomavirus infection. ciency virch 152:1673-6.

15 Breese PL, Judson FN, Penley KA, Douglas J Jr. AnaP human papillomavirus infection among homosexual andK bisexual men: prevalence of type-specific infection and association with human immunodeficiency virus. Sex. Transm Dis 1995;22:7-14.

16 Critchlow CW, Surawicz CM, Holmes KK, Kuypers $\mathrm{J}_{2}^{\text {जी }}$ Daling JR, Hawes SE, et al. Prospective study of higho grade anal squamous intraepithelial neoplasia in a cohor: of homosexual men: influence of HIV infection, immunosuppression and human papillomavirus infec- $-\bar{D}$ immunosuppression and human papillomavirus infec- $\frac{\vec{T}}{\text { tion. } A I D S}$ 1995;9:1255-62.

17 Williams AB, Darragh TM, Vranizan K, Ochia C, Moss AR, Palefsky JM. Anal and cervical human papillomavirus infection and risk of anal and cervical epithelial abnormalities in human immunodeficiency virus-infectedo women. Obstet Gynecol 1994;83:205-11.

18 Strickler HD, Rattray C, Escoffery C, Manns A, Schiffman MH, Brown C, et al. Human T-cell lymphotropic viruso type 1 and severe neoplasia of the cervix in Jamaica. Int fos Cancer 1995;61:23-6.

19 Castro K, Ward J, Slutsker L, Buehler JW, Jaffe HW Berkelman RL, et al. 1993 revised classification system for HIV infection and expanded surveillance case defini--

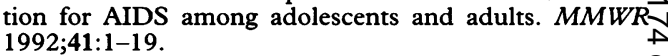

20 Ting Y, Manos MM. Detection and typing of genita human papillomaviruses. In: Innis $\mathrm{MA}$, Gelfand $\mathrm{DH}$, Sninsky JJ, White TJ, eds. PCR protocols and applications. San Diego: Academic Press, 1990:356-67.

21 Schiffman MH, Bauer HM, Hoover RN, Glass AG, Cadell DM, Rush BB, et al. Epidemiologic evidence showing that human papillomavirus infection causes most cervicako intraepithelial neoplasia. F Natl Cancer Inst 1993;85: 958-64.

22 Bosch FX, Munoz N, de Sanjose S, Izarzugaza I, Gili M, Viladiu $P$, et al. Risk factors for cervical cancer in Colombia and Spain. Int $\mathcal{f}$ Cancer 1992;52:750-8. 